ISSN: 0213-1854

\title{
Perspectivas en el estudio de la encuadernación artística: de los tratados generales a la web semántica
}

\section{(Perspectives on the study of fine bookbinding: from general treatises towards the semantic web)}

\author{
CARLOS DÍAZ REDONDO/ ELVIRA-JULIETA MiguÉLEZ-GONZÁLEZ \\ charlierdiaz@usal.es/ejulieta@usal.es \\ Biblioteca Diocesana de Granada/Universidad de Salamanca
}

Fecha de recepción: 1 de septiembre de 2016

Fecha de aceptación: 30 de septiembre de 2016

Resumen: Se presenta una revisión de las distintas metodologías aplicadas a lo largo del tiempo en el estudio de la encuadernación artística. Para ello, se recurre a la visión del arte ligatorio en tratados generales primero, y en manuales específicos después. Se atiende, asimismo, al cambio de paradigma motivado por el movimiento bibliófilo del siglo XIX, y a su repercusión en los posteriores estudios de los siglos XX y XXI. En último término, se analiza la importancia de las nuevas tecnologías en la investigación y difusión de la encuadernación artística, en medida que establecen vínculos con la web semántica y la musealización virtual del libro.

Palabras clave: Encuadernación artística. Fondo antiguo. Historia del libro. Musealización virtual. Patrimonio bibliográfico. Web semántica.

Abstract: A review of different methodologies applied in the study of fine bookbinding over time is presented. To this end, we draw upon the vision of binding art in general treatises first, and specific manuals then. The change in the paradigm motivated by the $19^{\text {th }}$ century bibliophile movement, and its impact on the subsequent $20^{\text {th }}$ and $21^{\text {st }}$ centuries studies, is discussed. Ultimately, we analyze the significance of new technologies in the research and dissemination of fine bookbinding, as they establish bonds with the semantic web and the virtual musealisation of books.

Key words: Bibliographic heritage; Fine bookbinding; History of the Book; Rare books; Semantic web; Virtual musealisation. 


\section{Introducción}

Desde la consolidación del libro en formato codex en el Egipto copto del siglo IV d. C. aproximadamente, su manufactura culmina inexorablemente con la encuadernación de la obra. De esta manera, el cuerpo del libro, formado por pliegos o cuadernillos cosidos y reforzados, se liga a través de diferentes procedimientos a una cubierta que está destinada a facilitar, por un lado, un buen manejo del objeto y, por otro, una mejor conservación. Esta cubierta, compuesta a su vez por un soporte en forma de tapas y un recubrimiento exterior, puede o no estar ornamentada mediante el empleo de distintas técnicas y bajo el influjo de diferentes corrientes artísticas. Partiendo de este hecho, el estudio de la encuadernación artística debe responder forzosamente a la exploración de los distintos métodos ligatorios, pero también al análisis y descripción de la ornamentación que la embellece.

Tradicionalmente, el acercamiento al estudio de la creación del libro se ha realizado siguiendo dos metodologías opuestas por completo. Por un lado, atendiendo a los conocimientos meramente prácticos recogidos en tratados generales y, más tarde, en manuales específicos. Por otro, a través de la observación directa del ejemplar.

La primera de estas metodologías se desarrolla hasta bien entrado el siglo XVIII y explora únicamente los aspectos técnicos del proceso, esto es, el procedimiento de montaje, el tipo de costuras realizadas, los materiales empleados, etc. El segundo método de estudio comienza a gestarse a partir del siglo XIX y, entre otras cosas, pone por primera vez de manifiesto la importancia de la ornamentación externa del libro.

\section{De los tratados de conocimientos generales a los manuales específicos de los siglos XVII y XVIII}

Las primeras alusiones escritas sobre el proceso de creación del libro que encontramos en la historia son sumamente vagas y aparecen recogidas en tratados de conocimientos generales. Esta escasez de fuentes documentales no resulta extraña si tenemos presente que, hasta la aparición de la imprenta, la producción del libro en la Edad Media está fuertemente marcada por un periodo de letargo, pues el acceso a la cultura estaba restringido a monasterios y centros religiosos, y un solo ejemplar podía tardar meses en ser copiado y encuadernado en el scriptorium.

En estos primeros textos, se referían únicamente operaciones de tipo secundario que no observaban la construcción del libro como un todo. Así, por ejemplo, en torno al año 1100, el monje benedictino Teófilo Presbítero, en su De Diversis Artibus, nos ponía en antecedentes sobre la fabricación de panes de 
Perspectivas en el estudio de la encuadernación artística: de los tratados...

oro para dorar los cantos de los libros, la preparación de colas y engrudos para reforzar, o el repujado de planchas de metal para cubrir tapas. En el siglo XIV, el tratado anónimo De arte illuminandi se ocupaba extensamente de la iluminación de manuscritos, aportando recetas para la composición de lacas y colores, y técnicas para crear relieves o aplicar y abrillantar pan de oro sobre soporte de pergamino. Unos años más tarde, en el primer tercio del siglo XV, el pintor gótico Cennino Cennini, en Il libro dell'arte, dedicaba un capítulo al modo de preparar y mezclar pigmentos para ejecutar iluminaciones. También el jesuita alemán Athanasius Kircher, en su Ars magna lucis et umbrae, estudió el color y sus juegos de luces y sombras, ocupándose sin pretenderlo de los patrones marmoleados que comenzarían a emplearse, por ejemplo, en los papeles de guarda.

Habrá que esperar, no obstante, hasta la aparición de los primeros manuales específicos del siglo XVII para comprender la encuadernación estudiada como un proceso unitario. Probablemente, el primero de todos ellos sea la Prescription et enseignement de la discrete et fameuse sciencie de la manufacture des relieurs de livres, escrita en 1612 por Anshelmus Faust, que ya contenía una descripción bastante fidedigna del procedimiento completo de composición del libro.

Entrando el siglo XVIII, concretamente en el año 1704, apareció un manuscrito que el tipógrafo Jacques Jaugeon, miembro de la Real Academia de Ciencias francesa, había comenzado a escribir en 1639. En su Description et perfection des arts et métiers (...) de relier les livres, Jaugeon no solamente aportaba una serie de pasos necesarios para la creación del libro, sino que además se detenía a explicar la construcción de los diferentes tipos y caracteres empleados en la imprenta.

A esta obra le siguieron otras. En 1741, por ejemplo, se publicó el que podemos considerar el primer manual completo de encuadernación, Der in aller beut zu Tag üblichen Arbeit wobl anweisende accurate Buchbinder und Futtermacher, de Christoph Ernst Prediger, en el que se detallaban minuciosamente las técnicas empleadas para la preparación de los materiales y sus precios en el mercado, así como los diferentes procesos y los tiempos por los que un conjunto de pliegos, un soporte en forma de tapas y un recubrimiento terminaban revelándose en una realidad individual en forma de libro.

Tras la obra de Prediger, en 1763 surgió el Traité de la reliure des livres, de JeanVincent Capperonnier de Gauffecourt. Y a su vez, basándose en estos primeros estudios y en la experiencia personal aportada por el encuadernador francés Le Monnier, en 1772 René Martin Dudin publicó L'art du relieur-doreur. Este manual de encuadernación, ilustrado originalmente por láminas de Ludovic Simoneau, estaba dirigido a profesionales del gremio y ha sido ampliamente reimpreso, 
reeditado y traducido a lo largo de los siglos. No en vano describe, ya de una manera gráfica y pormenorizada, tanto las técnicas de construcción y ornamentación del libro, como el taller y las herramientas de trabajo propias del maestro encuadernador.

\section{El nuevo paradigma del siglo XIX: la observación directa del ejemplar}

Durante las primeras décadas del siglo XIX, en pleno auge de la industrialización, siguieron apareciendo algunos manuales específicos, como los de Minshall (1811), Peignot (1823), LeNormand (1827) o Sabrel (1883), y otros textos cuanto menos particulares, caso de la obra del encuadernador Lesné (1820), poema didáctico escrito en verso en el que su autor realiza una compilación de técnicas ligatorias enriquecida con experiencias propias.

Aproximadamente a partir del último tercio del siglo, debemos considerar un giro radical en el estudio de la encuadernación, pues descendió la producción de textos exclusivamente técnicos, dirigidos a un lector muy concreto y con el único fin de formar libreros y encuadernadores, y se comenzó a aplicar una metodología totalmente diferente. Este nuevo paradigma, que se manifestará con mayor fuerza en los trabajos de los siglos XX y XXI, continuó en la línea de trabajo de la corriente de estudio técnica, pero viró el interés hacia el entendimiento del libro como un objeto único, focalizando la atención en los dos pilares que fundamentan esta unicidad: la rareza del ejemplar y la belleza, riqueza o singularidad de su encuadernación.

A partir de este momento, las obras empezaron a ilustrarse profusamente con reproducciones de las ornamentaciones externas de tapas, lomos, cantos y contracantos, unas veces mediante rudimentarios bosquejos, otras con grabados, mediante la técnica del frotis, o bien tardíamente con láminas fotográficas.

De esta manera, la observación directa del ejemplar, impulsada fundamentalmente por bibliófilos, coleccionistas, libreros, bibliotecarios y casas de subastas, permitió que las descripciones de las encuadernaciones pasasen de ser una mera relación de características referidas al proceso de construcción para comenzar a atender al estilo artístico de la ornamentación, con sus tres aspectos fundamentales.

Por un lado, los motivos ornamentales, que por sí mismos resultan a menudo insuficientes para caracterizar un estilo artístico, si bien permiten la realización de estudios comparativos de hierros, florones, planchas, etc., útiles para datar o determinar, por ejemplo, talleres y cecas de producción. Por otro lado, la composición, es decir, el modo en que esos motivos se combinan entre sí y forman estructuras que ayudan a reconocer un estilo decorativo en 
Perspectivas en el estudio de la encuadernación artística: de los tratados...

particular. Y, por último, el aspecto más complejo de los tres: la expresión, esto es, el reflejo de las sensaciones del artista que, aunque subjetivo por atender a la parte más emocional del ser humano, permite reconocer de forma objetiva el sello personal de determinados maestros en la manufactura de sus obras.

Este cambio en el tratamiento de la información relativa al arte ligatorio sentó el primer antecedente en la catalogación de encuadernaciones, pues es precisamente a través de los estilos, como en cualquier otra expresión artística, que tradicionalmente la ciencia las está clasificando.

Claros resultados de la aplicación de esta nueva metodología son, por poner algunos ejemplos, las obras de Gruel (1887), Bouchot (1888), Brunet (1891), Horne (1894) o Beraldi (1895).

\section{El auge de la bibliofilia y el coleccionismo de encuadernaciones}

No cabe duda de que la figura del bibliófilo fue durante el siglo XIX de suma importancia para el desarrollo de la historia del arte ligatorio. Gracias a los amantes del libro, las encuadernaciones artísticas mantuvieron altas cotas de valor en el mercado del arte y se consolidaron como objetos preciosos.

Y como resultado de aquel movimiento de bibliofilia, tratado por autores como Vindel (1934), o más recientemente por Sánchez Mariana (1993), se reunieron numerosas colecciones privadas de encuadernaciones, tanto europeas como americanas, que afortunadamente han llegado hasta nuestros días sin apenas dispersarse.

En España, contamos con colecciones magníficas, entre las que cabe señalar en primer término la del físico y médico vallisoletano Manuel Rico y Sinobas, adquirida por la Biblioteca Nacional de España en 1901 (Rubio de Urquía 2003), y que perfila, a través de sus más de mil tapas sueltas, la historia de la encuadernación desde el siglo XIV hasta el siglo XIX.

Es también importante el fondo reunido por Marcelino Menéndez Pelayo, director que fue de la Biblioteca Nacional de España y de la Real Academia de Historia, que donó los cerca de cuarenta mil volúmenes que integraban su colección a la ciudad de Santander, conservándose hoy en la biblioteca que lleva su nombre.

Destaca asimismo la colección del bibliófilo Manuel Pérez de Guzmán y Boza, I marqués de Jerez de los Caballeros, vendida en 1904 al millonario norteamericano Archer Milton Huntington (Rodríguez Moñino 1966), fundador de la Hispanic Society de Nueva York.

Es igualmente reseñable, por su altísimo interés histórico, la colección de las Casas de Osuna y del Infantado, con fondos de las bibliotecas de los marqueses de Santillana y de los condes-duques de Benavente. Adquirida por el Estado 
español, se integró en la Biblioteca Nacional de España en 1884, dos años después de haber fallecido su último propietario, Mariano Téllez-Girón y Beaufort-Spontin, XII duque de Osuna y XV del Infantado, que murió sin descendencia y acosado por las deudas tras haber dilapidado la fortuna familiar. Por diferentes avatares, una parte de esta colección se encuentra hoy distribuida entre diferentes bibliotecas universitarias españolas (Lilao Franca 2015), entre ellas, la Biblioteca General Histórica de la Universidad de Salamanca.

Gran interés artístico tiene la colección del editor y mecenas del arte, José Lázaro Galdiano, hoy propiedad de su Fundación, que comenzó a gestarse en las últimas décadas del siglo XIX en su palacete madrileño de Parque Florido, y que contiene todo tipo de estilos artísticos, desde encuadernaciones mudéjares hasta románticas, teniendo especial relevancia la muestra de encuadernaciones heráldicas (Yeves Andrés 2008).

Resulta significativa, por otro lado, la colección del jurista José Lameyer y González, bibliófilo y gran estudioso del arte ligatorio, formada por 249 obras técnicas sobre la encuadernación europea, repartidas en 327 volúmenes, algunos de ellos encuadernados por él mismo. Vendida en 1917 a Alfonso XIII, pasó a engrosar los ya importantísimos fondos de la Real Biblioteca, donde hoy se custodia.

Tampoco podemos dejar de mencionar la colección del bibliófilo bilbaíno Francisco Zabálburu y Basabe, que está formada en su mayoría por encuadernaciones de pergamino y románticas pero salpicada de algunas joyas de estilo mudéjar y heráldico (Llera Llorente 2005). Contiene además incunables procedentes de la biblioteca de los marqueses de Moya y de los condes de Heredia Spínola que fueron a parar a las estanterías de Zabálburu por haber contraído matrimonio su única hija con el conde de este mismo título.

Por último, es necesario reseñar la colección de la Fundación Bartolomé March Servera. Este fondo integra volúmenes de diversa procedencia, entre ellos parte de la biblioteca de la Casa de Medinaceli y de la Casa de Gor, y contiene gran número de encuadernaciones históricas que tocan prácticamente todos los estilos, desde el siglo XIV hasta el siglo XXI, con obras firmadas por encuadernadores contemporáneos de gran renombre, como Ramón Gómez, Emilio Brugalla o Antolín Palomino.

\section{La encuadernación artística durante el siglo XX: de las estanterías al espacio expositivo}

Más allá del creciente interés por la conformación y el estudio de las colecciones, el siglo XX trajo consigo numerosos trabajos relativos a las artes del libro y, por supuesto, grandes autores españoles que cultivaron la 
Perspectivas en el estudio de la encuadernación artística: de los tratados...

investigación sobre la encuadernación desde diferentes puntos de vista: la composición de catálogos, la recopilación de biografías de libreros y encuadernadores, etc. Entre los más destacados, podemos citar a Miquel y Planas (1913), Hueso Rolland (1934), Rico y Sinobas (1941), o Castañeda y Alcover (1958).

No obstante, el estudio del arte ligatorio español durante este periodo de tiempo no puede comprenderse sin atender a la exhaustiva producción literaria de López Serrano, quien llegara a ser directora de la Biblioteca de Palacio Real. Desde finales de los años 30 hasta prácticamente su fallecimiento, López Serrano enfocó sus esfuerzos no solamente a realizar labores de catalogación de fondo antiguo y encuadernaciones en diferentes bibliotecas, sino que su dilatada experiencia en el campo de la Biblioteconomía, y sobre todo su gestión al frente de la Real Biblioteca, propició la creación de una ingente cantidad de literatura. A ella se debe la denominación de algún estilo artístico de ornamentación, caso de los tipos populares (López Serrano 1972), o los primeros estudios de la obra de grandes encuadernadores españoles del siglo XVIII y XIX.

Y puesto que el estudio de la encuadernación está inseparablemente ligado al del libro, y éste al de las bibliotecas, es imprescindible citar a este respecto la extensa producción de Escolar Sobrino, antiguo director de la Biblioteca Nacional de España, que a lo largo de su vida generó un auténtico corpus teórico. Más allá de su manual por antonomasia, su Historia ilustrada del libro español contiene en tres tomos, editados en 1993, 1994 y 1996, una profunda revisión del libro, incluyendo numerosos trabajos sobre el arte ligatorio español.

Alrededor del último tercio del siglo XX, las encuadernaciones artísticas empezaron a abandonar sus estanterías, reclamando su condición de objeto valioso, merecedor de ser expuesto y admirado por el público. En consecuencia, comenzaron a realizarse exposiciones, suponiendo el primer acercamiento de la sociedad con la encuadernación artística más allá de los círculos bibliófilos, y generando la publicación de todo un repertorio de catálogos ilustrados. Generalmente, estos catálogos incluían entre sus páginas numerosos estudios y reproducciones fotográficas acompañadas de pequeños textos explicativos que, debido a la carencia de unas pautas normalizadas, describían las encuadernaciones de una manera somera y poco ordenada.

Hasta este momento, podemos afirmar que la investigación en torno al arte ligatorio tiene un tinte discontinuo, inconstante, un tanto anecdótico. Es a partir del siglo XXI cuando se observa una corriente seria de estudio, manifestándose un determinado método científico sujeto a procedimientos reproducibles, como la descripción y catalogación normalizada, o la incorporación de estos datos a distintos sistemas de información. 


\section{El siglo XXI: propuestas normalizadas de descripción y catalogación y el reto del entorno web}

El cambio de siglo supone en España un auge esperanzador en la investigación de la encuadernación artística. En los últimos años, han aparecido numerosos trabajos, generados como resultado de la investigación académica, publicados de manera muy dispersa en casas editoriales o revistas científicas ligadas por tradición al estudio de la Biblioteconomía y Documentación, las Artes Decorativas, la Filología y la Historia.

A este respecto, resulta imprescindible señalar la labor de la Asociación para el Fomento de la Encuadernación de Arte (AFEDA), que editó la revista Encuadernación de Arte desde 1993 hasta 2011, pudiéndose considerar en España una publicación de cabecera para los especialistas en esta materia. AFEDA fue asimismo responsable de la organización del Congreso Nacional sobre Bibliofilia, Encuadernación Artística, Restauración y Patrimonio Bibliográfico, que contó con tres ediciones, celebradas en 1999, 2004, y 2009.

No obstante, la mayor contribución del presente siglo al estudio del arte ligatorio han sido, con toda seguridad, las propuestas de modelos normalizados para la descripción y catalogación de encuadernaciones. Estas iniciativas, que van más allá de las reseñas escuetas que hasta ahora habíamos encontrado en los catálogos ilustrados, profundizan en el estudio de la encuadernación en distintos niveles, atendiendo a aspectos como la identificación de la obra, la técnica de construcción, los materiales empleados, el estilo artístico de ornamentación, la procedencia de la obra y sus marcas de propiedad, o el estado de conservación. Autores como Carpallo Bautista (2002), Clavería Laguarda (2006) o Miguélez González (2009) han aportado en sus trabajos distintas directrices descriptivas y pautas catalográficas, bebiendo todos ellos en gran medida de las fichas de control empleadas en laboratorios de restauración.

Otro de los aspectos más reseñables de la producción científica de los últimos años ha sido sin lugar a dudas el esfuerzo por clarificar los estilos artísticos de ornamentación. Su diferenciación, fundamental en la catalogación de cualquier encuadernación histórica, pasa por ser uno de los aspectos más complejos ante la gran cantidad de estilos que existen y las peculiaridades infinitas que cada uno de ellos presenta (Checa Cremades 2003).

$\mathrm{Y}$ a la luz de estos dos hechos, afloran nuevos fondos de encuadernaciones que hasta este momento eran desconocidos. Se trata, en muchos casos, de colecciones formadas al amparo de las funciones y actividades de diferentes instituciones, más privadas que públicas. Una gran parte de ellas pertenecen a la Iglesia Católica o a sus diversas órdenes religiosas, cuyos archivos y bibliotecas 
Perspectivas en el estudio de la encuadernación artística: de los tratados...

se presentan como un indudable nicho de estudio, pues aún guardan patrimonio bibliográfico virgen, en ocasiones sin controlar o catalogar.

Yendo más allá de la literatura tradicional, las nuevas tecnologías, y concretamente la llegada de Internet, han abierto múltiples vías de estudio. En este sentido, no hay duda de que nos encontramos ante un crecimiento progresivo del número de sistemas de información al servicio del acceso, estudio y difusión de la encuadernación y del libro antiguo (Herrera Morillas 2005).

Un claro ejemplo de este nuevo rumbo es la base de batos de encuadernación histórico-artística de la Real Biblioteca ${ }^{1}$, basada a su vez en iniciativas similares, como la de la British Library², la de la Biblioteca Nazionale Braidense de Milán ${ }^{3}$, la de la Biblioteca de Santa Genoveva de la Universidad de Sorbona Nueva ${ }^{4}$ o la de la Biblioteca Nacional de Francia ${ }^{5}$. El objetivo de todas ellas se centra en describir, estudiar y difundir la riqueza de las encuadernaciones atesoradas en estos centros pues se trata de propiciar la investigación sin renunciar a un necesario ánimo divulgativo.

Han comenzado a cubrirse igualmente campos de estudio que hasta hace algunos años parecían irrelevantes. Un buen ejemplo es el análisis de los datos de procedencia, indispensables en el campo de la encuadernación pues, si bien pueden parecer superfluos, ayudan al investigador no solamente a identificar a los antiguos dueños de los ejemplares, dotando al libro de una historia propia que lo enriquece, sino que además aportan luz acerca de aspectos que pueden determinar la identidad de los encuadernadores.

Derivados del estudio de marcas de propiedad como exlibris o superlibros, se están desarrollando proyectos innovadores, como las bases de datos de procedencias y antiguos poseedores de la Biblioteca General Histórica de la Universidad de Salamanca, la Biblioteca Histórica "Marqués de Valdecilla" de la Universidad Complutense, o del Centre de Recursos per a l'Aprenentatge i la

1 Vid. Patrimonio Nacional. Real Biblioteca. Base de datos de encuadernación bistórico-artística. Disponible en línea en: http://encuadernacion.realbiblioteca.es/ [Fecha de consulta: 1 de septiembre de 2016].

2 Vid. British Library. Database of bookbindings. Disponible en línea en: http://www.bl.uk/catalogues/bookbindings/ [Fecha de consulta: 1 de septiembre de 2016]. 3 Vid. Biblioteca Nazionale Braidense. Bookbinding. Disponible en línea en: http://www.braidense.it/bookbinding/index.html [Fecha de consulta: 1 de septiembre de 2016]. 4 Vid. Université Sorbonne Nouvelle, Bibliothèque Sainte-Geneviève. Reliures estampées à froid conservées à la Bibliothèque Sainte-Geneviève. Disponible en línea en: http://bsg-reliures.univparis1.fr/fr/accueil/ [Fecha de consulta: 1 de septiembre de 2016].

5 Vid. Bibliothèque nationale de France. Reliures. Disponible en línea en: http://reliures.bnf.fr/ [Fecha de consulta: 1 de septiembre de 2016]. 
Investigació de la Universitat de Barcelona, todas ellas volcadas además en el proyecto Provenance Information, del Consortium of European Research Libraries (CERL) $)^{6}$.

Especialmente trascendentes resultan asimismo iniciativas como la del grupo de investigación Bibliopegia, de la Universidad Complutense de Madrid, que en 2008 empezó a analizar la idea de crear una herramienta denominada Catálogo Colectivo de Encuadernaciones Artísticas (CCAE) 7 . Se pretendía crear una base de datos de tipo MySQL, con lenguaje de programación PHP y metadatos descriptivos DublinCore (DC), con el fin de gestionar descripciones, tanto textuales como gráficas y en un mismo formato, de encuadernaciones artísticas de diferentes fondos e instituciones (Carpallo Bautista, Tardón González y Uttranadhie 2014). Este proyecto, que resulta pionero al introducir directamente el arte ligatorio en la corriente de la web semántica, se encuentra hoy en día en fase beta y acumula ya descripciones de encuadernaciones procedentes de la Biblioteca Nacional de México, la Catedral de Toledo, la Real Academia de la Historia de España, la Fundación Casa de Alba, la Biblioteca Histórica de Madrid y la Biblioteca Histórica "Marqués de Valdecilla" de la Universidad Complutense.

Y si uno de los pilares fundamentales del estudio y de la difusión de la encuadernación han sido por excelencia las exposiciones físicas, en pleno apogeo de las herramientas de creación de contenidos digitales nos encontramos con un terreno excelente para la puesta en marcha de exposiciones virtuales. El modelo de musealización virtual, aún poco cultivado cuando hablamos de su aplicación al patrimonio bibliográfico, supone una serie de ventajas innegables con respecto a las exposiciones físicas que debemos tener presentes (Díaz Redondo, Hernández Olivera y Becedas González 2015).

En términos de gestión administrativa, el desarrollo de un producto virtual supone una agilidad incuestionable. Por poner un ejemplo, no es necesario solicitar órdenes ministeriales o autorizaciones de traslado de los materiales librarios.

En términos de producción y alcance, las exposiciones virtuales rompen con las limitaciones de tiempo, espacio y contenido. Una muestra puede estar accesible las 24 horas del día, los 365 días del año, desde cualquier rincón del mundo, ampliando enormemente el espectro del público potencial y

${ }^{6} \mathrm{Vid}$. Consortium of European Research Libraries. Provenance Information. Disponible en línea en: http://www.cerl.org/resources/provenance/main [Fecha de consulta: 1 de septiembre de 2016].

7 Vid. Universidad Complutense de Madrid. Catálogo Colectivo de Encuadernaciones Artísticas (CCAE). Disponible en línea en: http://freja.ccdoc.ucm.es:30080 [Fecha de consulta: 1 de septiembre de 2016]. 
Perspectivas en el estudio de la encuadernación artística: de los tratados...

permitiendo suministrar materiales complementarios de toda clase. Asimismo, el modelo virtual facilita al usuario un espacio interactivo en el que se proporciona no solamente la contemplación directa de una determinada encuadernación, sino que también hace factible el acceso al contenido íntegro digitalizado de la obra que cubre.

En términos económicos, es necesario recalcar el enorme abaratamiento de costes pues no es preciso, por ejemplo, tasar los ejemplares, asegurarlos, o contratar personal de seguridad para el espacio expositivo. Además, para el desarrollo técnico, es posible contar con softwares gratuitos de código abierto, que permiten poner en marcha una autentica infraestructura informática de calidad, adaptando un determinado sitio web a las necesidades específicas de un proyecto, facilitando las tareas de evaluación de resultados y produciendo una exposición que puede ampliarse y renovarse constantemente.

Finalmente, en términos de conservación, la difusión se realiza atendiendo a la perfecta preservación, pues las encuadernaciones exhibidas no sufren ni se deterioran con traslados o durante el proceso de montaje o desmontaje de la exposición.

En el caso de la musealización del libro antiguo, cuando se trata de un patrimonio en el que debe prevalecer el justo equilibrio entre la obligación de conservar y la de difundir, el modelo virtual es probablemente una de las mejores alternativas.

Y así lo ha entendido la comunidad científica, pues lentamente se está poniendo en práctica esta nueva forma de acercar la encuadernación a la sociedad, ahora sin necesidad de sacarla siquiera de su estantería. Sirvan como ejemplo las muestras virtuales de las bibliotecas de las universidades de Columbia $^{8}$, Indiana ${ }^{9}$, Miami $^{10}$, Monash $^{11}$ o Newfoundland ${ }^{12}$.

8 Vid. Columbia University, Library. Judging a book by its cover. Gold stamped publishers bindings of the 19th century. Disponible en línea en:

https://exhibitions.cul.columbia.edu/exhibits/show/bindings $[$ Fecha de consulta: 11 de septiembre de 2016].

${ }^{9}$ Vid. Indiana University, The Lilly Library. The fine Art of British Bookbinding. Disponible en línea en: http://www.indiana.edu/ liblilly/binding/virtual_exhibit.html [Fecha de consulta: $11 \mathrm{de}$ septiembre de 2016].

10 Vid. University of Miami, Library. Bound to Please: an exbibition of fine leather bindings. Disponible en línea en: http://scholar.library.miami.edu/bound/ [Fecha de consulta: 9 de septiembre de 2016].

11 Vid. Monash University, Library. Coloured Cloth Bindings. Disponible en línea en: https://www.monash.edu/library/collections/exhibitions/cloth/virtual-exhibition $[$ Fecha de consulta: 12 de septiembre de 2016].

12 Vid. Memorial University of Newfoundland, Queen Elizabeth II Library. Decorative Nineteenth Century Trade Bindings. Disponible en línea en: http:// collections.mun.ca/bindings/ 


\section{CARlos Díaz Redondo/Elvira-Julieta MiguÉlEZ-GonZÁLEZ}

En España, han sido fundamentalmente las bibliotecas universitarias quienes pueden considerarse pioneras. Véanse a este respecto las exposiciones de las bibliotecas de las universidades de Valencia ${ }^{13}$, Navarra ${ }^{14}$ o Zaragoza ${ }^{15}$. La Sección Nobleza del Archivo Histórico Nacional ha generado también una muestra virtual ${ }^{16}$, en la que se presenta una vista muy generalista de las encuadernaciones artísticas custodiadas en el centro, que parece dirigida a clarificar principalmente los estilos artísticos de ornamentación. Hasta el momento, la Biblioteca Nacional de España, a pesar del valioso fondo de que es depositaria, ha apostado únicamente por una exposición temática ${ }^{17}$ que versa exclusivamente acerca del libro mudéjar.

\section{Conclusiones}

El estudio de la encuadernación artística, considerada arte menor durante siglos, se ve ligeramente reflejado en los tratados de conocimientos generales hasta el punto que cada referencia hallada en ellos revela únicamente operaciones aisladas de tipo secundario. No será hasta la aparición de los primeros manuales específicos de los siglos XVII y XVIII cuando la creación del libro adquiera la entidad suficiente para ser tratada y analizada como un proceso unitario.

La observación directa del ejemplar, resultado del movimiento bibliófilo y coleccionista que germinó durante el siglo XIX, provocó un giro en el estudio del arte ligatorio sentando el precedente en la catalogación de encuadernaciones artísticas. Esta metodología se ha manifestado con claridad en los estudios de

[Fecha de consulta: 1 de septiembre de 2016].

13 Vid. Universidad de Valencia. Els vestits del saber: Enquadernacions mudèjars a la Universitat de València. Disponible en línea en: http://www.uv.es/cultura/v/docs/expvestitssaber.htm [Fecha de consulta: 11 de septiembre de 2016].

14 Vid. Universidad de Navarra, Biblioteca. Encuadernaciones artísticas. Disponible en línea en: http://www.unav.es/biblioteca/fondoantiguo/hufaexp09/hufaexp09p-01.html

[Fecha de consulta11 de septiembre de 2016].

15 Vid. Universidad de Zaragoza, Biblioteca General. Encuadernaciones artísticas en la Biblioteca General. Disponible en línea en:

https://biblioteca.unizar.es/biblioteca-general/encuadernaciones-introduccion

[Fecha de consulta: 11 de septiembre de 2016].

16 Vid. España, Ministerio de Educación, Cultura y Deporte. Encuadernaciones artísticas en el Archivo de la Nobleza. Disponible en línea en: http://www.mecd.gob.es/cultura-mecd/ca/areascultura/archivos/mc/encuadernaciones-artisticas/introduccion.html [Fecha de consulta: 1 de septiembre de 2016].

17 Vid. Biblioteca Nacional de España. Piel sobre tabla. Disponible en línea en: http://www.bne.es/es/Micrositios/Exposiciones/EncMudejares/ [Fecha de consulta: 8 de septiembre de 2016]. 
Perspectivas en el estudio de la encuadernación artística: de los tratados...

los siglos XX y XXI, resultando en último término en los primeros modelos normalizados de fichas catalográficas.

Si bien en la actualidad contamos con procedimientos y pautas para la catalogación de encuadernaciones, así como con herramientas digitales que permiten la implementación de bases de datos para la gestión colectiva de sus descripciones textuales y gráficas, existen aún muchos asuntos que demandan un análisis detenido. Tales son, por ejemplo, los diferentes estilos artísticos de ornamentación, que requieren aún una diferenciación clara por parte de los expertos y, especialmente, una urgente convención en su definición.

Si la investigación apunta necesariamente a la web semántica y a los datos enlazados, la difusión de la encuadernación artística pasa inevitablemente por su musealización virtual. Esta fórmula pone de manifiesto unas significativas ventajas frente a la producción de exposiciones físicas, visibles en términos de gestión administrativa, economía, contenido, acceso, interacción con el usuario, actualización, intemporalidad, versatilidad, medición de estadísticas, y conservación de los materiales originales.

\section{Referencias bibliográficas}

BERALDI, Henri, La reliure française du XIX siécle. Paris: Librairie Conquet, 1895.

BouchOt, Henri, Les reliures d'art à la Bibliothèque Nationale. Paris: Edouard Rouveyre, 1888.

BRUNET, Gustave, La reliure ancienne et moderne. Paris: Paul Daffis, 1878.

—, Études sur la reliure des livres et sur les collections des bibliophiles célèbres. Bordeaux: Vve. de Moquet, 1891.

CAPRONNIER DE GAUFFECOURT, Jean-Vicent; SMYTH, Elaine B. (ed.), Traité de la relieure des livres: a bilingual treatise on bookbinding. Austin: W. Thomas Taylor, 1987.

CARPallo BAUTiSTA, Antonio, Análisis documental de la encuadernación española: repertorio bibliográfico, tesauro y ficha descriptiva. Madrid: AFEDA, 2002.

CARPAllo BAUTISTA, Antonio; TARDÓN GONZÁlez, Eugenio Rubén; UtTRANADHIE MarTín, Alberto, "Desarrollo del Catálogo Colectivo de Encuadernaciones Artísticas (CCEA)". En: Interinformación: XI Jornadas Españolas de Documentación: 20, 21 y 22 de mayo de 2009, Auditorio Palacio de Congresos de Zaragoza, 2009, pp. 233-240.

CASTAÑEDA Y ALCOVER, Vicente, Ensayo de un diccionario biográfico de encuadernadores españoles. Madrid: Imprenta Editorial Maestre, 1958.

CheCa CRemades, José Luis, Los estilos de encuadernación: (siglos III d.J.C.-XIX). Madrid: Ollero \& Ramos, 2003. 
Clavería LAgUARDA, Carlos, Reconocimiento y descripción de encuadernaciones antiguas. Madrid: Arco, 2006.

Díaz Redondo, Carlos; Hernández Olivera, Luis (dir.); BeCEDAS GONZÁLEZ, Margarita (dir.), En el principio fue la palabra: exposición virtual para la Biblioteca General Histórica de la Universidad de Salamanca. Salamanca: Universidad de Salamanca, Facultad de Geografía e Historia, 2015.

DuDIN, René Martin, Arte del encuadernador. Madrid: Ollero \& Ramos, 1997.

ESCOLAR SOBRINO, Hipólito, Historia ilustrada del libro español: Los manuscritos. Madrid: Fundación Germán Sánchez Ruipérez, 1993.

- Historia ilustrada del libro español: De los incunables al siglo XVIII. Madrid: Fundación Germán Sánchez Ruipérez, 1994.

- Historia ilustrada del libro español: La edición moderna. Siglos XIX y XX. Madrid: Fundación Germán Sánchez Ruipérez, 1996.

FAUST, Anshelmus, Prescription et enseignement de la discrete et fameuse sciencie de la manufacture des relieurs de livres. Bruselas: Bibliotheca Wittockiana \& Fl. Tulkens, 1987.

GRUEL, Léon, Manuel historique et bibliographique de l'amateur de reliures. Paris: Gruel \& Englemann, 1887.

HERRERA MORILLAS, José Luis, "Encuadernaciones antiguas: sistemas para su estudio, difusión y acceso en Internet". En "Scire", 11, no 1 (enero-junio de 2005), pp. 123-135.

HORNE, Herbert, The binding of books. London: K. Paul, Trench, Trübner \& Co., 1894.

HuESO ROLLAND, Francisco, Exposición de encuadernaciones españolas. Siglos XII al XIX: catálogo general ilustrado. Madrid: Sociedad Española de Amigos del Arte, 1934.

JAUGEON, Jacques, Description et perfection des arts et métiers, des arts de construire les caractères, de graver les poinçons de lettres, de fondre les lettres, d'imprimer les lettres et de relier les livres, par Monsieur Jangeon, de l'Accadémie roale des Sciences. 1704.

LENORMAND, Louis-Sebastien, Manuel du relieur dans toutes ses parties. Paris: Rouret, 1827.

LESNE, La reliure, poëme didactique en six chants: précédé d'une idée analytique de cet art, suivi de notes historiques et critiques... Paris: chez Lesné, 1820.

LILAO FRANCA, Óscar, "La biblioteca de la casa de Osuna en las bibliotecas universitarias españolas: marcas de procedencia". En: Pecia Complutense, 2015, año $12, \mathrm{n}^{\circ} 22$, pp. 34-44.

Llera LlORENTE, María Teresa, "El estudio de los fondos de la Biblioteca Francisco Zabálburu”. En: Revista General de Información y Documentación, 2005, vol. $15, \mathrm{n}^{\circ} 2$, pp. 103-128. 
Perspectivas en el estudio de la encuadernación artística: de los tratados...

LÓPEZ SERRANO, Matilde, La encuadernación española. Breve historia. Madrid: ANABA, 1972.

MiguÉLEZ GONZÁLEZ, Elvira Julieta, La encuadernación artística de la Biblioteca Histórica de la Universidad de Salamanca: estilos y técnicas. Salamanca: Universidad de Salamanca, Departamento de Biblioteconomía y Documentación, 2009.

MINSHALL, N., The whole art of bookbinding, containing valuable recipes for sprinkling, marbling, colouring... Oswestry: N. Minshall, 1811.

MIQUEL Y PLANAS, Ramón, El arte hispano-árabe en la encuadernación. Barcelona: Miquel-Rius, 1913.

PEIGNOT, Gabriel, Manuel de bibliophile ou traité du choix des livres. Dijon: chez Victor Lagier, 1823.

PREDIGER, Christoph Ernst, Der in aller heut zu Tag üblichen Arbeit wobl anweisende accurate Buchbinder und Futtermacher. Anspach: zufinden in der Poschischen Hofbuchhandlung, 1772.

RiCO Y SiNOBAS, Manuel, El arte del libro en España. Madrid: Escelicer, 1941.

RODRÍGUEZ MOÑINO, Antonio, Catálogo de la biblioteca del marqués de Jerez de los Caballeros: reimpreso por primera vez en facsimil, precedido de una biografía del gran bibliófilo. Madrid: Librería para Bibliófilos, 1966.

RUBiO DE URQUíA, Guadalupe, "La colección de encuadernaciones Rico y Sinobas de la Biblioteca Nacional”. En: Encuadernación de Arte, 21 (2003), pp. 35-37.

SABREL, M., Manual completo del encuadernador, teórico y práctico: descripción de las máquinas y procedimientos modernos y antiguos. Barcelona: Manuel Saurí, 1883.

SÁNCHEZ MARIANA, Manuel, Bibliófilos españoles: desde los orígenes hasta los albores del siglo XX. Madrid: Biblioteca Nacional, 1993.

VINDEL, Francisco, Los bibliófilos y sus bibliotecas desde la introducción de la imprenta en España hasta nuestros dias. Madrid: [s.n.], 1934.

YEVES ANDRÉS, Juan Antonio, Encuadernaciones heráldicas de la biblioteca Lázaro Galdiano. Madrid: Ollero \& Ramos: Fundación Lázaro Galdiano, 2008. 